\title{
ADAPTIVE ARRAY SUPERRESOLUTION :CONTROL-LOOP NOISE
}

\author{
Mostafa A. Bahie-Eldin
}

\begin{abstract}
In recent years, considerable effort has been spent in developing high adaptive array superresolution techniques. This paper presents a perturbation expansion method named "two-variable technique" to derive an expression for the output power of the adaptive null-steering antenna array considering the statistical weight fluctuations. This array utilizes Howells-Applebaum type adaptive weight. Thus, in turn, the paper presents the difference between the assumed and actual adaptive array signal processing response of different high resolution techniques. These techniques are based on maximum-likelihood method, adaptive-angular response, and thermal noise algorithm. By evaluating the model performance with different signal-to-noise ratios, number of antennas, and different signal arrival angles for selected test cases, we gain insights into the sensitivity of the signal estimation problem to model uncertainty.
\end{abstract}

\section{INTRODUCTION}

Array signal processing has been a very attractive research field for several decades motivated by the demand for sensor, radar, radio, seismic, speech, and other signal processing in applications of electronic surveillance, telecommunications, astronomy, geography, oceangeography, and bio-medical science. In the last decade, adaptive antenna processing has found promienence in the problem of detecting multiple narrow-band sources and estimating their direction of arrival (DOA's) [1]. The adaptive antenna pattern has nulls which may be located arbitrarily close together in terms of beamwidth, without violáting any physical principle, such as the Rayleigh criterion on the diffraction limit of our aperture. Yet, because the nulls have served to locate many signal sources wihin a beamwidth, one may describe this as a "superresolution" pattern. The superresolution algorithms based on adaptive array have been introduced. Among of them;, Maximum-likelihood method (MLM) [2], adaptive angular response (AAR) [3], and Gabierl's thermal noise algorithm (TNA) [1].

For the direction of arrival wave estimation, an extensive literature exists to deal with "closed-loop" adaptive techniques [4-7]. By "closed-loop", we

Assoc. Prof. in Communication Dept., MTC, Cairo, EGYPT. 
mean techniques in which the signal at the output of the 1 inear combiner is used to adjust the weights of the combiner output. By this technique, it is considered a two-step signal estimation procedure. In the first step, the direction of arrivals (DOA's) of all the signals is estimated using one of the different techniques mentioned above. In the second step, the estimated signals are obtained as a linear combination of the array outputs, with weights which are computed from the estimated DOA's. The linear combiner is designed so that the given DOA's are completely estimated.

The adaptive weight elements in the adaptive control loop are not fixed in the steady-state but they have components that vary in a random manner. This occurs because of the random components of the received processes. The random components of the adaptive weights give rise to additional noise in the output signal which is of interest as one measure of how great a penalty is incurred by the adaptive processor's ability to maintain precisely optimum weight settings. The weight adjustment algorithms of these adaptive weight elements are described by time-varying stochastic differential equations.

Past researchers [8-10] gave a complete derivation for the resulting additional loop noise in the array output when the input signal consists of the interference signal only. A general expression of the output power of the adaptive antenna at the steady-state for any number of the incident signals is derived. The problem of detecting multiple narrow-band sources and estimating their directions of arrival by means of different superresolution methods taking into consideration the adaptive weight fluctuations is investigated. These methods will be comparatively studied in this work. The study will discuss the effect of the signal-to-noise ratio (SNR), number of antennas (N) and angle of arrival (AOA).

\section{PROBLEM FORMULATION}

The superresolution array considered in this wrok is shown in Fig.1. The source signals are received by a linear array with $\mathrm{N}$ different antenna elements at $d$ a half wavelength apart. The received vector signal, $X(t)$, is multiplied by the vector adaptive weight, $w(t)$, where $w(t)=\left[w_{1}(t), w_{2}(t)\right.$, . ..., $\left.w_{N}(t)\right]^{T}$ and superscript $T$ denotes the vector transpose. The array output signal $\mathrm{y}(\mathrm{t})$ is the weighted sum of the array input signals,

$$
y(t)=w^{T}(t) x(t)
$$

This is followed by a processor computing the final superresolution array output according to its definition (see Fig. 1). The adaptive weights are computed from the Howells-Applebaum (H-A) algorithm [11]. This wel1-known 
algorithm is simple, robust, requires no data storage, and may be implemented in either analog or digital form. Its adaptive vector weight is defined by the following stochastic differential equation

$$
\tau_{i} d W(t) / d t=-\left(I+g X(t) X^{+}(t)\right) w(t)+g S
$$

where $I$ is NxN identity matrix, $S$ is the steering vector in the adaptive loops and $g$ and $\tau_{i}$ are respectivly the loop gain and the time constant of the low-pass filter in (H-A) loop. Furthermore, dagger ${ }^{+}$denotes the complex conjugate transpose.

Eq. (2) is a stoachastic linear differential equation with stochastic input $X(t)$ and the stoachastic solution $W(t)$. The statistics of the input signal will be modeled as sample functions of stationary, uncorrelated, and zero mean random processes. The total power of the array output $y(t)$ is defined as the expectation of the magnitude-squared array output signal. The result is

$$
\langle\mathrm{P}(\mathrm{t})\rangle=\left\langle|\mathrm{y}(\mathrm{t})|^{2}\right\rangle=<\left[\mathrm{w}^{+}(\mathrm{t}) \mathrm{X}(\mathrm{t}) \mathrm{x}^{+}(\mathrm{t}) \mathrm{w}(\mathrm{t})\right)>
$$

where $<>$ denotes the ensemble average or the expectation.

A superresolution array "pattern" can be viewed from a practical implementation point as a map of the output (residual) power at an array, with the capability of adapting to the signal source distribution, plotted as a function of the angle, after the adaptation has reached the steady state. The

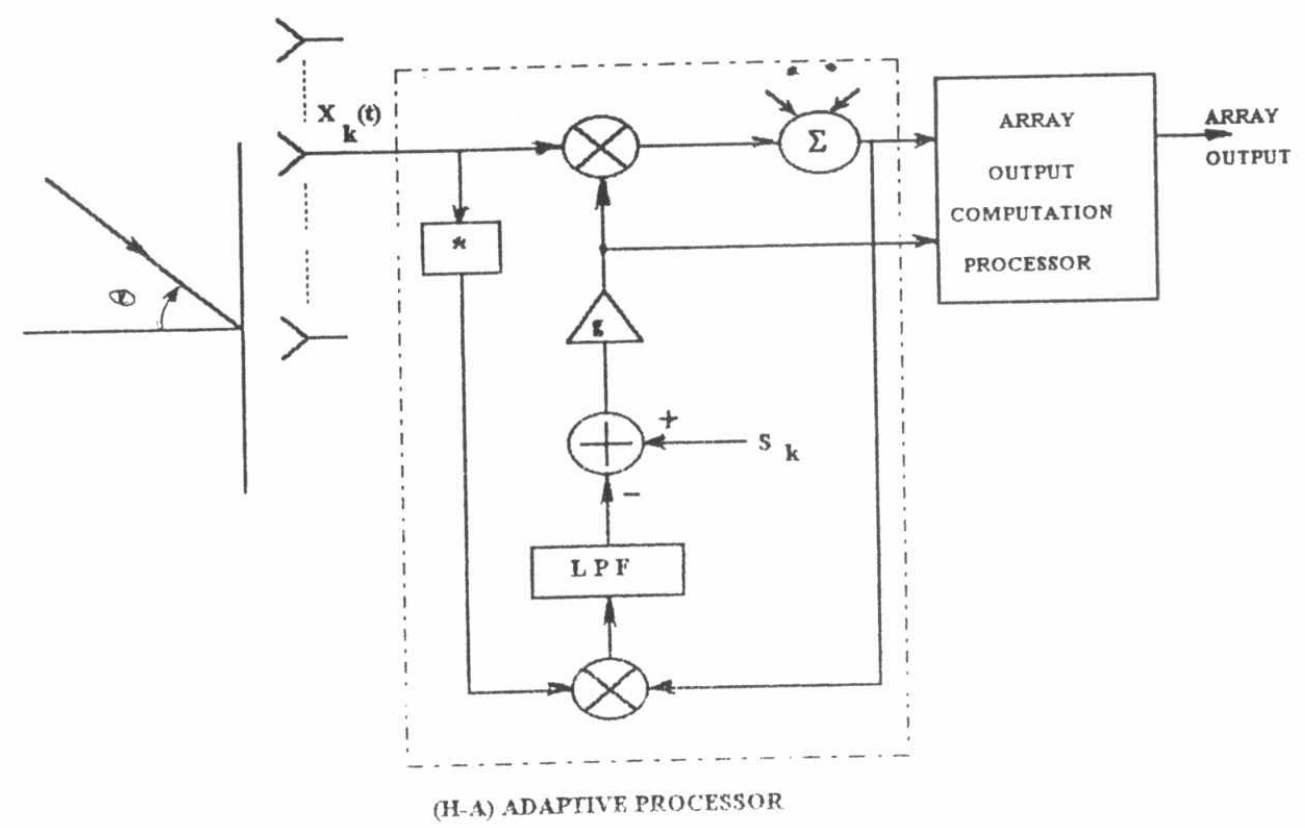

Fig.1 Superresolution array configuration considered in this paper 
superresolution adaptive arrays considered in this work are the maximum likelihood method (MLM), adaptive antenna response (AAR), and thermal noise algorithm (TNA).

The standard methodology used to obtain tractable approximate solutions for (3) is to find the average weights by ignoring the effects of fluctuations about the mean. The resulting variance of the weights about the mean values is presumably smal1. By other words, we can assume that the mean of the weights is constant with probability one. This is equivalent to assuming that the stochastic weights are statistically independent of the input signals. Taking into consideration this assumption, (3) becomes

$$
<\mathrm{P}(\mathrm{t})>=\mathrm{W}^{+}(\mathrm{t})<\mathrm{X}(\mathrm{t}) \mathrm{X}^{+}(\mathrm{t})>\mathrm{W}(\mathrm{t})
$$

We call (4) the approximate mean power equation. This equation has been used in most analyses. One of the objectives of this paper is to obtain an expression for the output power with releasing this assumption, and in turn applying the result into the espressions of the superresolution.

The MLM was developed by McDonough [3] for the processing of seismic data; however, as he pointed out, the technique has applications in radar, sonar, and radio astronomy. The maximum likelihood spatial spectrum is identical to the use of a zeroth-order mainbeam directional gain constraint in adaptive arrays, where the "spatial spectrum" would be estimated by the output residual power $\mathrm{P}$ from the optimized adapted array weights. Under the zeroth-order gain constraint, we require $\mathrm{S}^{+} \mathrm{w}_{0}=1$, where $\mathrm{w}_{\mathrm{O}}$ is the optimized weight vector. Upon sweeping the steering vector $S$ for a given input signal, the power $P$ will estimate the spatial spectrum. The superresolution function $G_{M L M}(\theta)$, for this case, is defined as the inverse of the residual power P,

$$
\mathrm{G}_{\mathrm{MLM}}(\theta)=\frac{1}{\mathrm{P}(\theta)}
$$

The AAR can be simply defined as the map of the distribution of signal sources obtained through a special nonlinear processing of the set of cross correlations of the voltages at the terminals of the elements of a planar or linear array. The AAR spectrum normalizes the output power of the array to a thermal noise term $\left(P_{n}(\theta)\right)$. The AAR spectrum is given by [4]

$$
G_{\text {AAR }}(\theta)=\frac{P(\theta)}{P_{n}(\theta)}
$$

Gabriel's thermal noise possesses the desirable characteristics of Maximum 
Likelihood Method (MLM). It also produces sharp narrow peaks, and retains a low residual background. In TNA to obtain superresolution of the external signal sources, the reciprocal of the adapted thermal noise power output of the array is plotted versus the angle of arrival (scan direction). This definition is in accordance with the viewpoint of AAR of an adaptive phase array. The TNA utilizes a spectral estimation scheme using only the thermal noise term [1]. It is characterized by the denominator term of the AAR:

$$
\mathrm{G}_{\mathrm{TNA}}(\theta)=\frac{1}{\mathrm{p}_{\mathrm{n}}(\theta)}
$$

Here, we are interested in clarification the difference in the steady-state performance of different techniques using $\mathrm{H}-\mathrm{A}$ adaptive superresolution array taking into consideration the effect of weight fluctuations for an environment which consists of signal sources and additive receiver noise. We assume that the signals and the thermal noise signals at the array input are uncorrelated zero mean signals. The receiver noises in each element are independent of each other and are Gaussian signals with zero mean, and variance $\alpha^{2}$.

\section{ANALYSIS OF THE LOOP NOISE POWER}

To evaluate the additional output power containing the stochastic weight fluctuations, we must develop the differential equation for the power. This is because we need to compute the total power (coherent and incoherent). Let us begin by differentiating the instantaneous output power. This gives

$$
\begin{aligned}
& \frac{d P(t)}{d t}=x^{+}(t) \frac{d w(t)}{d t} w^{+}(t) x(t)+x^{+}(t) w(t) \frac{d w^{+}(t)}{d t} x(t) \\
& +\frac{d x^{+}(t)}{d t} w(t) w^{+}(t) x(t)+x^{+}(t) w(t) w^{+}(t) \frac{d x(t)}{d t}
\end{aligned}
$$

Substituting Eq. (2) in Eq. (8) gives

$$
\tau \text { i } \frac{d P(t)}{d t}=-2(g Q(t)+1) P(t)+2 g R e\left\{S^{+} M(t) W(t)\right\}+f(t)
$$

where $\operatorname{Re}\{\}$ designates the real part of the complex quantity,

$$
f(t)=2 \tau_{i} \operatorname{Re}\left\{\frac{d x^{+}(t)}{d t} H(t) x(t)\right\},
$$


and

$$
H(t)=w(t) w^{+}(t)
$$

The inner product of the input signal is $Q(t)=X^{+}(t) X(t)$

Let us assume that the input signal vector, $X(t)$, has a bandwidth $B$. The variation of the signal will be exhibited explicitly by writing $X(t)$ as $X\left(t / T_{s}\right)$ where $T_{s}=1 / B$. Now, making a change of independent variable in (9) from $\mathrm{t}$ to $\tau=\mathrm{t} / \mathrm{T}_{s}$, we obtain

$$
\frac{\mathrm{dP}(\tau)}{\mathrm{d} \tau}=-2 \varepsilon(\mathrm{gQ}(\tau)+1) \mathrm{P}(\tau)+2 \varepsilon \operatorname{gRe}\left\{\mathrm{S}^{+} \mathrm{M}(\tau) \mathrm{W}(\tau)\right\}+\mathrm{f}(\tau)
$$

where $\varepsilon=\mathrm{T}_{s} / \tau_{\mathrm{i}}$. The parameter $\varepsilon$ represents the ratio of the open circuit bandwidth of LPF in the feedback loop to the bandwidth of the input process. In most practical arrays, where the loop bandwidth is small compared to the signal bandwidth, $\varepsilon$ is a very small positive quantity. Introducing $\varepsilon<<1$ into (12) sets the problem up for a solution using perturbation analysis.

The solution of (12) will be a function of the independent variable $\tau$ and the parameter $\varepsilon$. Since the regular perturbation expansion in terms of $\varepsilon$ for this solution does not converge for large $\tau$ (steady-state), which is our main interest, we will employ the two-variable technique $[12,13]$ to obtain an expression for $\mathrm{P}$ valid for all $\tau$. This kind of expansion perturbation is called singular perturbation. Eq. (12) for $P(\tau)$ involves $W$, and the equation for $W$ is in [14].

The two-variable perturbation analysis postulates that $W$ and $P$ are such a function of the fast variable $\tau$ and a slow variable $\bar{\tau}=\varepsilon \tau$. So, we write $W$ as $W(\tau, \bar{\tau}, \varepsilon)$, and $\mathrm{P}$ as $\mathrm{P}(\tau, \bar{\tau}, \varepsilon)$. Next these functions are expanded in a power series in $\varepsilon$ as follows:

$$
\begin{aligned}
& W=W(\tau, \bar{\tau}, \varepsilon)=\sum_{m=0}^{\infty} \varepsilon_{m}^{m} W_{m}(\tau, \bar{\tau}) \\
& P=P(\tau, \bar{\tau}, \varepsilon)=\sum_{m=0}^{\infty} \varepsilon_{m}^{m} P_{m}(\tau, \bar{\tau})
\end{aligned}
$$


The introduction of another time variable $\bar{\tau}$ increases the number of independent variables and by using the chain rule, the total derivative with respect to $\tau$ can be written as a sum of partial derivatives

$$
\frac{\mathrm{d}}{\mathrm{d} \tau}=\frac{\partial}{\partial \tau}+\varepsilon \frac{\partial}{\partial \bar{\tau}}
$$

Now, by substituting Eqs. (13), (14) and (15) into Eq. (12) of the power and by equating coefficients of equal powers of $\varepsilon$, equations for $P_{m}, m=0,1,2$, $\ldots \ldots$ can be obtained. The equations for $w_{m}$ are given in [14] and will not be repeated here. The equations for $\mathrm{P}_{1}, \mathrm{P}_{2}$, and $\mathrm{P}_{3}$ are:

$\varepsilon^{o}: \frac{\partial P_{o}(\tau, \bar{\tau})}{\partial \tau}=f_{0}(\tau, \bar{\tau})$

$$
\begin{aligned}
\varepsilon^{1}: \frac{\partial \mathrm{P}_{1}(\tau, \bar{\tau})}{\partial \tau}=-\frac{\partial \mathrm{P}_{0}(\tau, \bar{\tau})}{\partial \bar{\tau}}-2(\mathrm{gQ}(\tau)+1) \mathrm{P}_{0}(\tau, \bar{\tau}) \\
+2 \operatorname{gRe}\left\{\mathrm{S}^{+} \mathrm{M}(\tau) \mathrm{W}_{\mathrm{o}}(\tau, \bar{\tau})\right\}+\mathrm{f}_{1}(\tau, \bar{\tau}) \\
\varepsilon^{2}: \frac{\partial \mathrm{P}_{2}(\tau, \tau)}{\partial \tau}=-\frac{\partial \mathrm{P}_{1}(\tau, \bar{\tau})}{\partial \bar{\tau}}-2(\mathrm{gQ}(\tau)+1) \mathrm{P}_{1}(\tau, \bar{\tau}) \\
+2 \operatorname{gRe}\left\{\mathrm{S}^{+} \mathrm{M}(\tau) \mathrm{W}_{1}(\tau, \bar{\tau})\right\}+\mathrm{f}_{2}(\tau, \bar{\tau})
\end{aligned}
$$

and

$$
\mathrm{f}_{m}(\tau, \bar{\tau})=2 \operatorname{Re}\left\{\frac{\mathrm{dX}^{+}(\tau)}{\mathrm{d} \tau} \mathrm{H}_{\mathrm{m}}(\tau, \bar{\tau}) \mathrm{X}(\tau)\right\} \quad, \mathrm{m}=0,1,2, .
$$

The solution of the above equations in conjunction with the solution for the weight vector as given in [14] contains arbitrary functions of time variables $\tau$ and $\bar{\tau}$. The basic idea is the formation of rules for the construction of the successive terms of the expansion (14) in such a way that it does not diverge for large values of $\tau$ (steady-state). It is required that no bounded terms which grow linearly with $\tau$ (secular terms) can be permitted in the solution.

Following [14] and solving the above equations, one can obtain equations for 
$<\mathrm{P}_{\mathrm{O}}(\tau, \bar{\tau})>$ and $\left\langle\mathrm{P}_{1}(\tau, \bar{\tau})>\right.$. The procedure is as follows: the solution of (16) is considered first. It can be written as:

$$
\mathrm{P}_{\mathrm{o}}(\tau, \bar{\tau})=\mathrm{P}_{\mathrm{o}}(\bar{\tau})+\int_{0}^{\tau} \mathrm{d} \tau^{\prime} \mathrm{f}_{\mathrm{o}}\left(\bar{\tau}^{\prime}, \bar{\tau}\right)
$$

where $P_{0}(\bar{\tau})$ is a constant of integration. It is a function of the slow variable $\bar{\tau}$ only since the derivative in (16) is with respect to $\tau$. Next to solve (17) for $\mathrm{P}_{1}(\tau, \bar{\tau})$, we substitute (20) into (17) and intergate with respect to $\tau$ treating $\bar{\tau}$ as a fixed quantity.

The two-variable perturbation method imposes a condition on $\mathrm{P}_{1}(\tau, \bar{\tau})$ so that the terms which are growing linearly in $\tau$ (secular terms) cancel out. In other words, as $\tau$ approaches $\omega$, the term $\mathrm{P}_{1}$ must be bounded. This ensures that the first order perturbation is less than the zeroth order one. Thus, we write

$$
\lim _{\tau \rightarrow \infty} \frac{P_{1}(\tau, \bar{\tau})}{\tau}=0 \quad \bar{\tau}-\text { fixed }
$$

Following the same procedure as shown in [14], the total output array power is

$$
<\mathrm{P}>\simeq<\mathrm{P}_{\mathrm{O}}>\left(1+\frac{\mathrm{sg}^{2}}{(\mathrm{~g}<\mathrm{Q}>+1)} \overline{<(\mathrm{Q} \Delta \mathrm{Q})>}\right)
$$

where $\mathrm{P}_{\mathrm{O}}$ is the output power of the antenna array when neglecting the weight fluctuations, and

$$
\overline{\langle Q \Delta Q\rangle}=\lim _{\tau \rightarrow \infty} \frac{1}{\tau} \int_{0}^{\tau} \mathrm{d} \tau_{0}^{\tau^{\prime}} \int_{0}^{\mathrm{d}} \mathrm{d}\left\langle\left\{\mathrm{Q}(\tau)\left(\mathrm{Q}\left(\tau^{\prime}\right)-\langle\mathrm{Q}\rangle\right)\right\}\right\rangle
$$

This represents the fourth moment of the input processes of the antenna array.

From (22), we may obtain an understanding of the effects of the input signals statistics and the control loop gain on the additional loop noise. An examination of the expression for $\langle\mathrm{P}\rangle$ as given by (22) shows that setting $\varepsilon$ to zero, we find $\langle\mathrm{P}\rangle$ equals $\left.<\mathrm{P}_{\mathrm{O}}\right\rangle$. This term corresponds to the standard result which is obtained by using the common assumption as presented by most analyses 
while the second term $\left(\mathrm{L}_{\mathrm{a}}\right)$ of $(22)$ is a new result. This result expresses the additional loop output power caused by the weight fluctuations. This term as will be shown in the next section plays an important role for detecting multiple signals calculated by (5), (6), and (7). The two-variable expansion technique used, here, to derive $(22)$ is very general, and powerful method. The results obtained by this method is completely consistent with exact analytic calculations which have been derived for Guassian input signal [8], and special random process called Continuous State Jump-Markov Process (CSJMP) [10].

\section{NUMERICAL RESULTS}

In this section, we present examples that utilize the diffrent superresolution algorithms. The following discussion will give excellent insight to all concerned.

\section{CASE I: ONE INCIDENT SIGNAL PLUS THERMAL NOISE}

Here, we assume that a single complex Gaussian narrow-band signal with a bandwidth $B$ is incident on the array. Thus, the received signal vector at the array input may be written as

$$
X(t)=s(t) \xi+N(t)
$$

where $s(t)$ is the complex envelope of the external signal source, and $N(t)$ is $N x 1$ column receiver noise vector whose $k$-th element is $n_{k}(t)$. The array delay propagation vector of the incident signal is

$$
\xi=\left(1, e^{-i \phi}, . . . . \quad, e^{-i(N-1) \phi}\right)^{T}
$$

where $\phi$ is the phase-delay for the signal and is expressed as

$$
\phi=(2 \pi \mathrm{d} / \lambda) \sin \theta
$$

with $\lambda$ is the wavelength of the incident planar wavefront and $\theta$ is the signal arrival angle measured from broadside. To calculate the total output power (22), we need to determine $\langle Q\rangle$, and $\overline{\langle Q \Delta Q\rangle}$. For stationary input process, the results are

$$
<\mathrm{Q}>=\mathrm{N} \sigma^{2}(1+\alpha)
$$

and

$$
\overline{\langle Q \Delta Q>}=\mathrm{N}^{2} \sigma^{4}\left(1+\alpha^{2}+2 \alpha / \mathrm{N}\right) / 4
$$


with $\alpha$ is the signal-to-noise ratio.

Substituting the resultants (27), and (28) into (22), the expression for the total output power is obtained. Employing this result in (5), (6), and (7) the different superresolution techniques are evaluated. Using the results of (27) and (28), the second term $\mathrm{L}_{\mathrm{a}}$ of (22) is calculated. Table 1 summarizes the values of this term obtained for the different values of SNR and $\mathrm{N}$, and with two values of $\varepsilon(\varepsilon=0.05$, and 0.1$)$.

TABLE 1. values of $\mathrm{L}_{\text {a }}$ for single incident signal

\begin{tabular}{|c|c|c|c|c|}
\hline \multirow{2}{*}{ SNR (dB) } & \multicolumn{2}{|c|}{$\mathrm{N}=7$} & \multicolumn{2}{c|}{$\mathrm{N}=11$} \\
\cline { 2 - 5 } & $\varepsilon=0.05$ & $\varepsilon=0.1$ & $\varepsilon=0.05$ & $\varepsilon=0.1$ \\
\hline 0 & 0.0058 & 0.0117 & 0.0103 & 0.0206 \\
5 & 0.0186 & 0.0373 & 0.0314 & 0.0628 \\
10 & 0.0731 & 0.1462 & 0.1187 & 0.2374 \\
15 & 0.2595 & $\underline{0.5191}$ & $\underline{0.4128}$ & $\underline{0.8257}$ \\
20 & $\underline{0.8568}$ & 1.7136 & 1.3518 & 2.7037 \\
25 & 2.7484 & 5.4968 & 4.3246 & 8.6491 \\
\hline
\end{tabular}

From Table 1, we can notice the weight fluctuations effect. For $\mathrm{N}=11$ and $\varepsilon=0.05$, and 0.1 this effect is sensible for SNR greater than $15 \mathrm{~dB}$, while for $N=7$, and $\varepsilon=0.05$, the effect of the weight fluctuations is at the value of SNR greater than $20 \mathrm{~dB}$, and for $\varepsilon=0.1$, this effect is at value of $\mathrm{SNR} \geqslant 15 \mathrm{~dB}$.

Now, we present several numerical examples. First, Fig. 2 illustrates an adapted pattern of the superresolution gain (SRA) for the three techniques (MLM, AAR, and TNA) otained from an 7 element linear array with a far field signal $20-\mathrm{dB}$ source located at $0^{\circ}$ off broadside. The element spacing is half-wavelength. The figure has six curves computed from (5), (6), and (7), three for neglecting the weight fluctuations effect, and other three for considering thses fluctuations. Fig. 2-a is for $\varepsilon=0.1$, while, Fig. 2-b is for $\varepsilon=0.05$.

Several interesting points can be made. Among of them; the pattern peak aligns perfectly with the location of the source incidence for all different algorithms. The three techniques are inherently capable of superresolution. The weight fluctuations degrade the superresolution capability. this effect increases with the increase of the value of $\varepsilon$. The important observation made is that the AAR performance is improved by considering these weight fluctuations. The explanation behind is that the expression of AAR is 


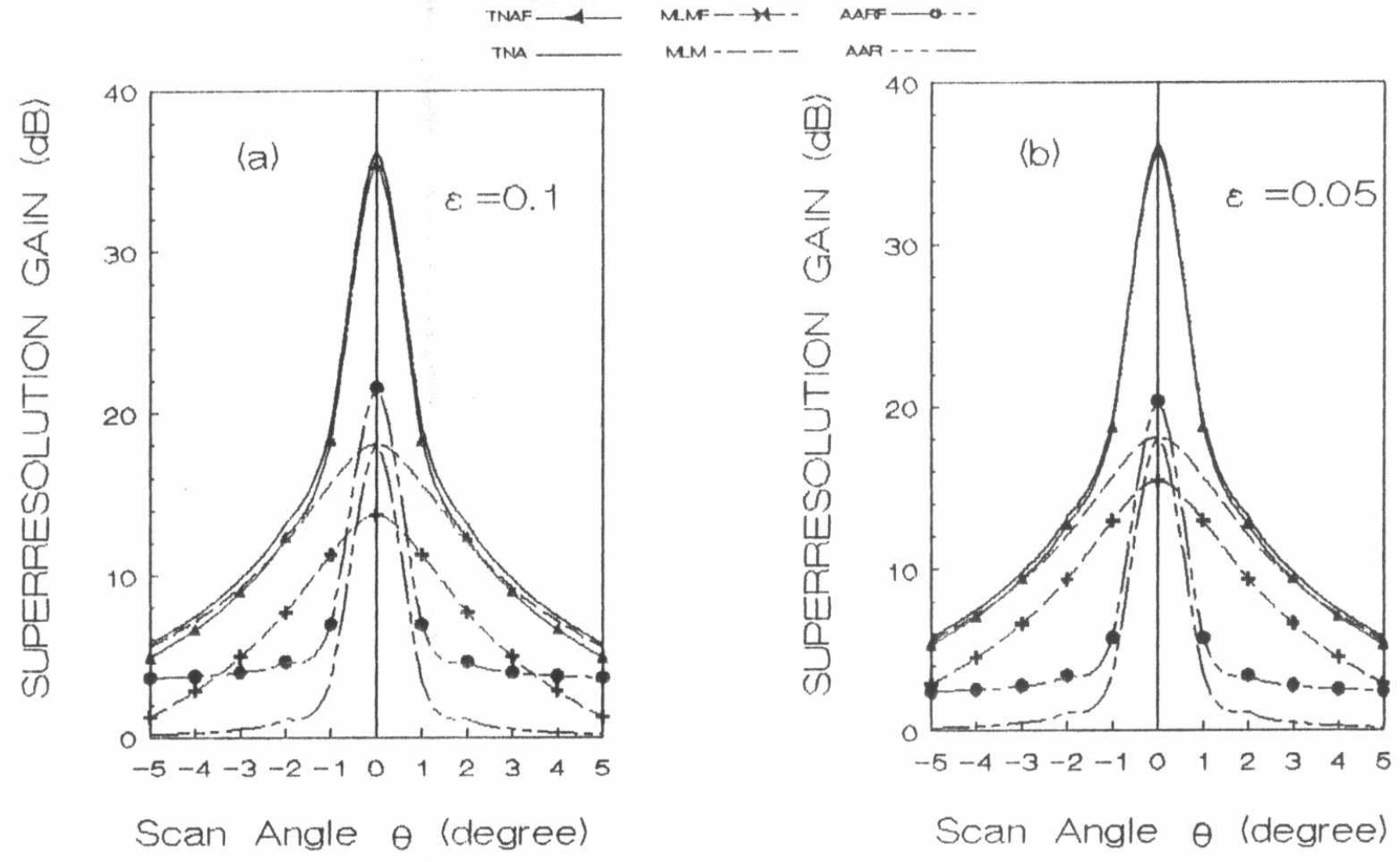

Fig.2 SRA versus $\theta$ for one signal with $d / \lambda=.5, \theta=0$, and $\alpha=20 d B$ normalized with respect to the power of thermal noise.

CASE I I :TWO INCIDENT SIGNALS PLUS THERMAL NOISE

In this case, the received signal can be represented as:

$$
\mathrm{X}(\mathrm{t})=\mathrm{s}_{1}(\mathrm{t}) \xi_{1}+\mathrm{s}_{2}(\mathrm{t}) \xi_{2}+\mathrm{N}(\mathrm{t})
$$

where $s_{1}(t)$ and $s_{2}(t)$ are the complex envelope of the two Gaussian narrow-band incident signals, and $\xi_{1}$ and $\xi_{2}$ are the array delay propagation vectors for the two signals arrived at the angles $\theta_{1}$ and $\theta_{2}$, respectively. They are defined in similar manner as for $\xi(10)$. For this case, the values of $<Q>$, and $\overline{\langle Q \Delta Q\rangle}$ are

$$
<\mathrm{Q}>=\mathrm{N} \sigma^{2}\left(1+\alpha_{1}+\alpha_{2}\right)
$$

and

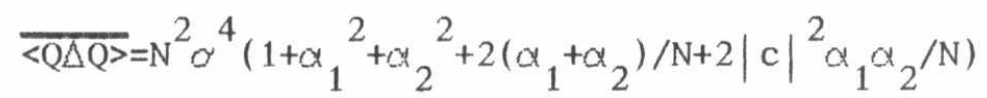


with $\alpha_{1}$ and $\alpha_{2}$ are the signal-to-noise ratios for the two incident signals, respectively, and $\mathrm{c}$ is the spatial correlation coefficient between the two incidents signals. It is given as $\mathrm{c}=\xi^{+}{ }^{+} \xi$.

Putting (30) and (31)in equation (22), the total output power can be calculated and the second term of (22) is obtained. Putting the result of (22) in (5), (6), and (7) we obtain the expressions for different superresolution algorithms .

Using the results of (30) and (31), the second term $\mathrm{L}_{\mathrm{a}}$ of (22) is calculated. Table 2 summarizes the values of this term obtained for $\alpha_{2}=10 \mathrm{~dB}$, and for different values of $\alpha_{1}, \mathrm{~N}$, and two seperation angles $\Delta \theta\left(\Delta \theta=3^{\circ}\right.$, and $\left.5^{\circ}\right)$ with two values of $\varepsilon(\varepsilon=0.05$, and 0.1$)$.

TABLE 2 values of $\mathrm{L}_{\mathrm{a}}$ of two incident signals

\begin{tabular}{|c|c|c|c|c|c|c|c|c|}
\hline \multirow{3}{*}{$\operatorname{SNR}_{1} \ell \mathrm{d} \mathrm{B}$} & \multicolumn{4}{|c|}{$N=7$} & \multicolumn{4}{|c|}{$\mathrm{N}=11$} \\
\hline & \multicolumn{2}{|c|}{$\varepsilon=0.05$} & \multicolumn{2}{|c|}{$\varepsilon=0.1$} & \multicolumn{2}{|c|}{$\varepsilon=0.05$} & \multicolumn{2}{|c|}{$\varepsilon=0.1$} \\
\hline & $\Delta \theta=3^{\circ}$ & $\Delta \theta=5^{\circ}$ & $\Delta \theta=3^{\circ}$ & $\Delta \theta=5^{\circ}$ & $\Delta \theta=3^{\circ}$ & $\Delta \theta=5^{\circ}$ & $\Delta \theta=3^{\circ}$ & $\Delta \theta=5^{\circ}$ \\
\hline 0 & 0.1503 & 0.1354 & 0.3006 & 0.2709 & 0.2882 & 0.2143 & 0.5764 & 0.4286 \\
\hline 5 & 0.2871 & 0.2467 & 0.5743 & 0.4933 & 0.584 & 0.3839 & 1.1681 & 0.7678 \\
\hline 10 & 0.5702 & 0.4813 & 1.1405 & 0.9626 & 1.1739 & 0.7385 & 2.3477 & 1.4770 \\
\hline 15 & 1.0093 & 0.8661 & 2.0187 & 1.7323 & 2.014 & 1.3211 & 4.0280 & 2.6422 \\
\hline 20 & 1.7653 & 1.5879 & 3.5305 & 3.1757 & 3.2902 & 2.4379 & 6.5805 & 4.8758 \\
\hline 25 & 3.7241 & 3.5322 & 7.4482 & 7.0643 & 6.4043 & 5.4851 & 12.809 & 10.970 \\
\hline
\end{tabular}

From Table 2, we can notice group of observations. The weight fluctuations effect increases with increase of $\mathrm{N}$, SNR, and decrease of angle seperation. For $\mathrm{N}=7$, and $\varepsilon=0.05$ the value of $\mathrm{L}_{\mathrm{a}}$ is effective for $\alpha_{1} \geqslant 10 \mathrm{~dB}$ at $\Delta \theta=3^{\mathrm{O}}$, and $\Delta \theta=5^{\circ}$, while for $\varepsilon=0.1$, the effective value of $L_{a}$ is at $\alpha_{1} \geqslant 5 \mathrm{~dB}$. For $N=11$, and $\varepsilon=0.05$, the effective value of $L_{a}$ is at $\alpha_{1} \geqslant 5 \mathrm{~dB}$ for $\Delta \theta=3^{\circ}$ and at $\alpha \geqslant 10 \mathrm{~dB}$ for $\Delta \theta=5^{\circ}$, while for $\varepsilon=0.1$ the effective value of $L_{a}$ is at $\alpha_{1} \geqslant 0 \mathrm{~dB}$.

Figures 3 and 4 show the plot of superresolution gain for the three algorithms 

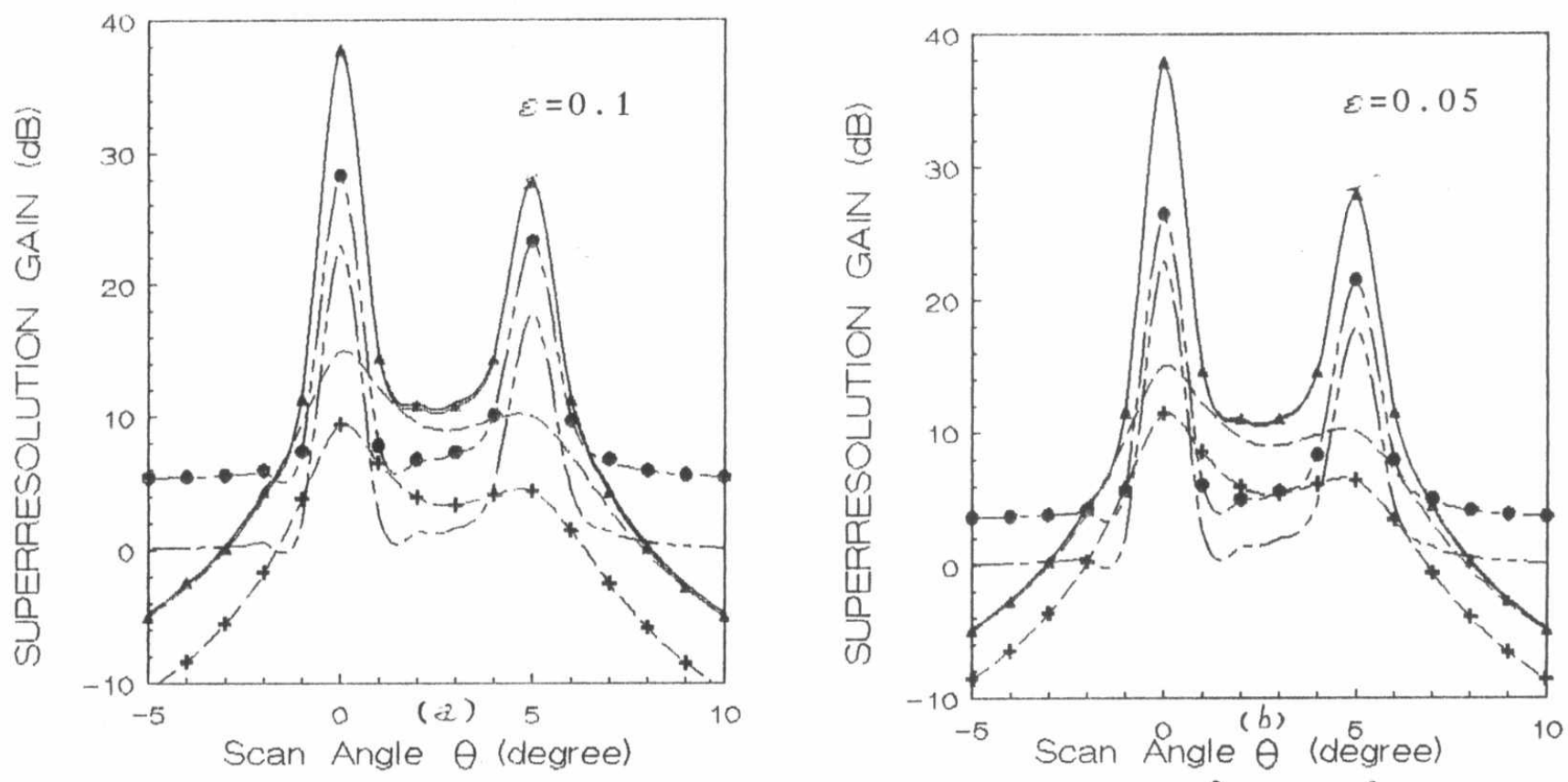

Fig. 3 SRA versus $\theta$ for two signals with $d / \lambda=.5, N=11, \theta=0^{\circ}$ and $\theta=5^{\circ}$,

and $\operatorname{SNR}_{1}=15 \mathrm{~dB}$, and $S \mathrm{NR}_{2}=10 \mathrm{~dB}$
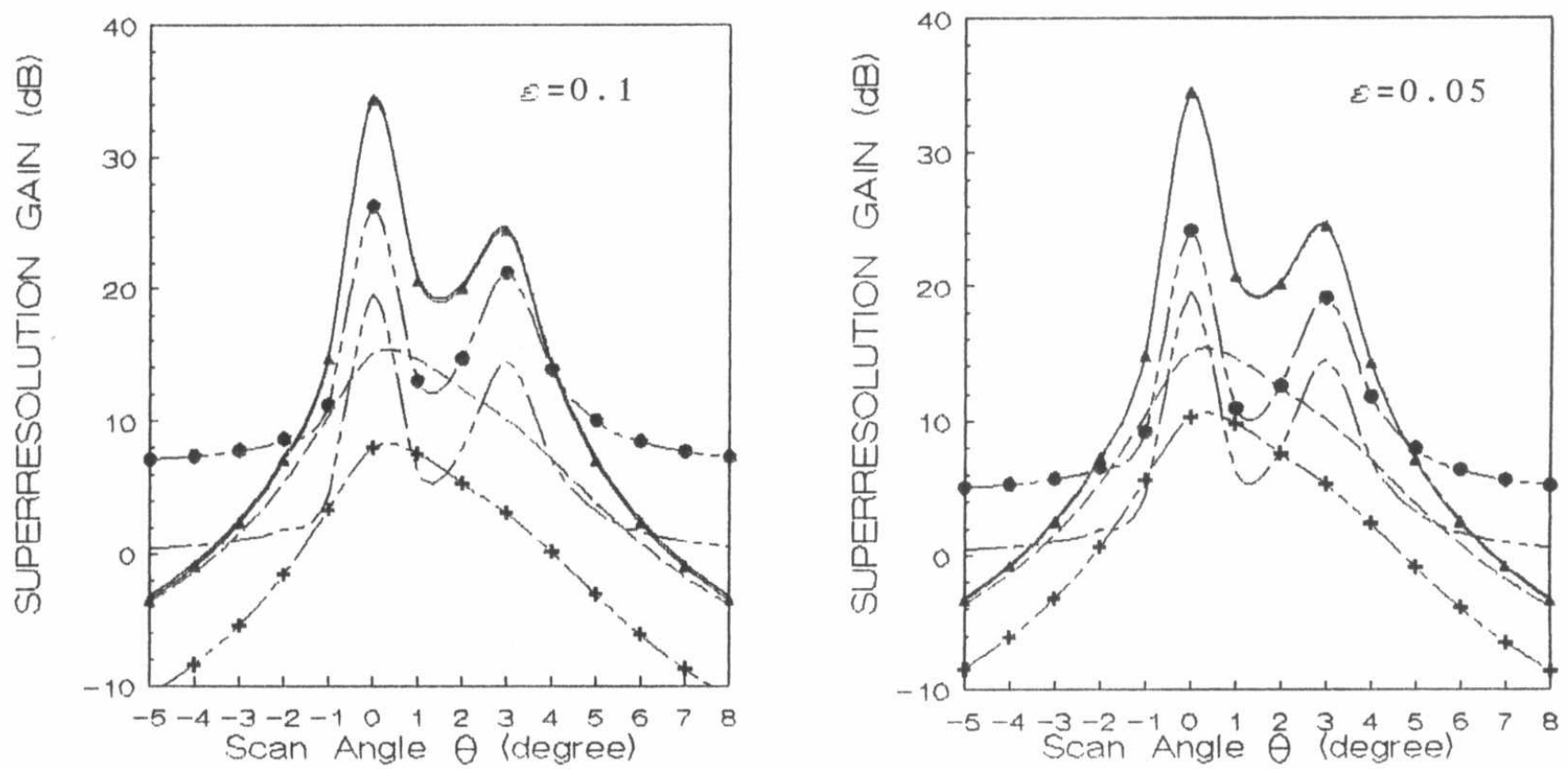

Fig. 4 SRA versus $\theta$ for two signals with $d / \lambda=.5, N=11, \theta=0^{\circ}$ and $\theta=3^{\circ}$. 
(MLM, AAR, AND TNA) versus the scan angle $\theta$ where the element spacing is half-wavelenght, $\quad \mathrm{N}=11, \theta_{1}=0^{\mathrm{O}}, \Delta \theta=5^{\circ}$ and $3^{\mathrm{O}}$ and $\alpha_{1}=15 \mathrm{~dB}$, and $\alpha_{2}=10 \mathrm{~dB}$. The figures have six curves computed from (5), (6), and (7), three for neglecting the weight fluctuations effect, and other three for considering thses fluctuations. Fig. 3 is for $\Delta \theta=5^{\circ}$ with $3-\mathrm{a}$ for $\varepsilon=0.1$, and $3-\mathrm{b}$ for $\varepsilon=0.05$, while Fig. 4 is for $\Delta \theta=3^{\circ}$ with 4 -a for $\varepsilon=0.1$, and $4-b$ for $\varepsilon=0.05$. Note that the two superresolution peaks align perfectly with the locations of two sources. Another important point to note is that smaller the angle seperation increases the superresolution capability degradation. Third point is that the weight fluctuations degrade the system performance except the AAR alogrithm.

\section{CONCLUSION}

A perturbation expansion method named "two-variable technique" has been employed to investigate the effect of the adaptive weight fluctuations of actual adaptive array superresolution techniques. These techniques that utilize H-A adaptive algorithm are MLM, AAR, and TNA. Two different cases have been studied. In both cases, it is shown that the number of antenna elements (N), signal-to-noise ratios, angle seperation, and control loop gain develop an additional loop noise at the antenna output power. This, in turn, degrades the superresolution capabilities. Table 1, and Table 2 summarize the values of the additional term obtained by the weight fluctuations effect on the output power for different N, SNRs, and angle seperation. The important observation made is that the AAR performance is improved by considering these weight fluctuations.

\section{REFERENCES}

[1]Gabriel, W. F.,"Spectral analysis and adaptive array superresolution techniques" proceedings IEEE, Vo1. 68, No. 6, June 1980.

[2]McDonough, R. N., Application of the maximum-1ikelihood method and maximum-entropy method to array processing. In S. Haykin (Ed.), Nonlinear methods of spectral analysis, New York: Springer-Verlag, 1979, pp. 209-211.

[3]Borgiotti, G. V., and Kaplan, L. J., "Superresolution of uncorrelated interference sources by using adaptive array techniques", IEEE Trans. on Antennas and Propagat., Vol. AP-27, No. 11 Nov. 1979.

[4]Friedlander, B., and Weiss, A. J., "Effects of model errors on waveform estimation using the MUSIC algorithm" IEEE Trans. on Signal Processing, Vol. 42, No. 1, pp. 147-155 Jan. 1994. 
[5]Friedlander, B., and Porat, B., "Performance analysis of a null steering algorithm based on direction of arrival estimation" IEEE Trans. Aoust. Speech, Signal Processing, Vol. 37, NO. 1, pp. 461-466, Jan. 1989.

[6]Li, F., and Vaccaro, R. J., "Unified analysis for DOA estimating algorithms in array signal processing," Signal processing, Vol. 25, pp. 147-169, Nov. 1991.

[7]Bahie-Eldin, M. A., "Comparative performance of different adaptive superresolution array algorithms" IEEE MILCOM 94, vol. 3, October, 1994.

[8]Brennan, L. E., Pugh, E. L., and Reed, I. S., "Control loop noise in adaptive array antennas", IEEE Trans. Aerosp. Electron. Syst. vol. AES-17, pp. 254-262, Mar. 1971.

[9]Berni, A. J., "Weight jitter phenomena in adaptive array control loops," IEEE Trans. Aerosp. Electron. Syst., vol. AES-13, pp. 335-361, July 1977.

[10]Gerlash, K., Application of probabilistic methods in adaptive array, D.Sc. Dissertation, GWU, Feb. 19, 1981.

[11]Applebaum, S. P., "Adaptive arrays" IEEE Trans. on Antennas and Propagat. Vol. AP-24, No. 4, Sept. 1976.

[12]Cole,J. D., Perturbation Methods in Applied Mathematics, Blaisdell Publishing Co., 1968.

[13]Papanicolaou, G., and Keller, J. K., "Stochastic Differential Equation with Applications to Random Harmonic Oscillators and Wave Propagation in Random Media", SIAM J. App1. Math. Vo1. 21, No. 2, Sept. 1971.

[14]Lang, R.,Bahie-Eldin, M. A., and Pickholtz, R. L., "Stochastic Effects in Adaptive Null-Steering Antenna Array Performance", IEEE journal on selected area in communication, vol. JSAC-3, No. 5, Sept. 1985, pp. 767-778. 